\title{
Quality over Quantity: A Case Based Review of HDL Function and Dysfunction
}

\author{
Suleiman Suleiman, $M B, B C H, B A O^{*}(\mathrm{D}, J J$ Coughlan, $M B, B C H, B A O, M R C P I$ \\ and Vincent Maher, MD, FRCPI, FESCFACC
}

Department of Cardiology, Tallaght University Hospital, Ireland

*Corresponding author: Suleiman Suleiman, $M B, B C H, B A O$, Department of Cardiology, Tallaght University Hospital, Tallaght, D24, Dublin, Ireland

\begin{abstract}
High density lipoprotein cholesterol (HDL) has traditionally been considered athero-protective and has been associated with a reduced risk of atherosclerotic cardiovascular disease (ASCVD). This has led to an approach of increasing HDL levels in high risk patients. However, due the complexity of the HDL particle and its diverse role in inflammation and immunity, this simple approach may be detrimental to cardiovascular health. Recent analysis of large population cohorts has established a U-shaped association between HDL and adverse events, further complicating our understanding of the clinical significance of HDL.

In this manuscript, we present two cases of patients with high levels of $\mathrm{HDL}$ and atherosclerotic vascular disease. $\mathrm{HDL}$ function is discussed and some recommendations as to how physicians should interpret and manage lipid abnormalities in the setting of a raised HDL. We will finish by discussing future directions of research in this undoubtedly complex area.
\end{abstract}

\section{Introduction}

High density lipoprotein cholesterol (HDL) has historically been considered as "good cholesterol' and has been associated with a reduced risk of atherosclerotic cardiovascular disease (ASCVD) [1]. The Framingham study identified a strong positive association between low HDL levels and coronary heart disease [2]. This led to widespread belief that HDL was a modifiable risk factor for ASCVD and thus treatments were developed to increase HDL levels to obtain cardio-protection [3]. Understanding HDL's role in ASCVD is however more perplexing than initially expected. HDL is a complex, heterogenous molecule, with a varied proteome [4].
A large number of proteins involved in inflammation, complement regulation, and innate immunity are physiologically bound to HDL [5]. These reinforce the concept that HDL evolved as a component of the innate immune system and is reflected in its pleiotropic functions in the body [6]. With its constant remodeling via enzymes and tissues, various subclasses, structural diversity, interactions, and functionality, HDL has a larger role in health and disease [7]. This inverse correlation between raised levels of HDL and ASCVD is not necessarily causal [8] and numerous studies have failed to show reduced ASCVD risk with elevation of HDL [9-11]. Contemporary data has highlighted that higher levels of HDL may be detrimental to cardio-vascular health, demonstrating a unique U-shape association between raised HDL and ASCVD risk [12] in men. This paradoxical rise in ASCVD risk is likely driven by several reasons including genetic mutation, the concept of HDL dysfunction and inflammation. This had led to an increasing interest in quantifying HDL function and though the reverse cholesterol transport process (RCT) has been given primacy with regard to the proposed mechanistic relationship between HDL and ASCVD, HDL also demonstrates anti-inflammatory, anti-apoptotic, anti-oxidative and vaso-protective properties [13-16].

\section{Case 1}

Our first case is a 54-year-old female who was referred from our colleagues in age related healthcare. She had presented to their service with non-specific neurological symptoms and was subsequently diag- 
nosed with a seizure disorder. MRI of her brain had demonstrated some non-specific white matter foci which were felt to be most likely microvascular in origin. Her lipid profile was deranged with a total cholesterol of $7.1 \mathrm{mmol} / \mathrm{L}$ (Normal: < 5.0), Triglycerides of $0.8 \mathrm{mmol} / \mathrm{L}$ (Normal $<1.7 \mathrm{mmol} / \mathrm{L}$ ), low density lipoprotein (LDL) of $3.7 \mathrm{mmol} / \mathrm{L}$ (Normal < 3.0) and high density lipoprotein (HDL) of $3.0 \mathrm{mmol} / \mathrm{L}$ (Normal > 1.2). Her referring physician had noted that she had encountered side effects with several different statins and therefore referred this lady to our clinic for consideration of PCSK9 inhibitor therapy. The referral letter specifically mentioned that she had a 'reassuringly high HDL'. Her background medical history included vitamin B12 deficiency, hypothyroidism secondary to a partial thyroidectomy in 1972, Epilepsy and she was an ex-smoker with a 15 pack year history. Her current medications included: Levothyroxine 125 mcg once daily, levetiracetam 500 mg once daily, aspirin $75 \mathrm{mg}$ once daily and hydroxycobalamin 1000 $\mathrm{mcg} / \mathrm{ml}$ every three months. We performed a comprehensive biochemical risk marker screen. This revealed a glycosylated hemoglobin of $38 \mathrm{mmol} / \mathrm{L}$ (Normal $<42$ $\mathrm{mmol} / \mathrm{LO}$, c-reactive protein (CRP) of 1.4 (Normal < 5), homocysteine of $9.4 \mathrm{umol} / \mathrm{L}$ (Normal $=0-15$ ), Lipoprotein (a) of $482.9 \mathrm{nmol} / \mathrm{L}$ (Normal < 72), TSH of $0.16 \mathrm{mU} / \mathrm{L}$ (0.3-4.2) and Free T4 of $18 \mathrm{pmol} / \mathrm{L}$ (12-22). As mentioned previously, her MRI Brain was suggestive of microvascular disease and her carotid doppler ultrasound (CDUS) revealed mixed type 3 and 4 plaques bilaterally. On review of her "statin intolerance", we discovered that her lipid profile was described as 'not controlled' on atorvastatin $40 \mathrm{mg}$ once daily in 2007. In 2009 she switched to rosuvastatin but experienced agitation and discontinued therapy. She then trialled simvastatin and experienced muscular stiffness, predominantly in her hands. We started this lady on atorvastatin $20 \mathrm{mg}$ once daily with co-enzyme q10 supplementation. Lercanidipine $10 \mathrm{mg}$ once daily was also started as she was noted to be hypertensive on 24 hour blood pressure monitor. Our patient experienced no issues on statin therapy and her LDL improved to $1.9 \mathrm{mmol} / \mathrm{L}$ (Baseline: $3.7 \mathrm{mmol} / \mathrm{L}$ ), with an increase in HDL to $3.7 \mathrm{mmol} / \mathrm{L}$ (Baseline: 3.0 $\mathrm{mmol} / \mathrm{L})$.

\section{Case 2}

Our second case is a 57-year-old female who was referred to our risk factor clinic with "a total cholesterol level of $7.6 \mathrm{mmol} / \mathrm{L}$ despite lifestyle measures". Her baseline lipid profile showed a total cholesterol of 7.6 $\mathrm{mmol} / \mathrm{L}$. A triglyceride level of $0.7 \mathrm{mmol} / \mathrm{L}, \mathrm{LDL}$ of 4.3 $\mathrm{mmol} / \mathrm{L}$ and $\mathrm{HDL}$ of $3.01 \mathrm{mmol} / \mathrm{L}$. She had no medical history of note, was an ex-smoker with a 5 pack year history. She drank 4 units of alcohol per week and was active, exercising five times per week. Her BMI was normal at 21 and she was normotensive in clinic (119/69 $\mathrm{mmHg}$ ). Her family history was notable for a father who was diagnosed with angina in his thirties and who un- derwent coronary artery bypass grafting in his sixties. Of her ten siblings, three were currently taking statin therapy although none had suffered from a myocardial infarction or stroke. She was taking no medications. Additional screening blood-work revealed normal $\mathrm{HbA} 1 \mathrm{c}$, homocysteine and lipoprotein (a) levels. Carotid Doppler ultrasound revealed soft plaque at the level of the carotid bulb bilaterally. We started this lady on atorvastatin 20 mg once daily and continue to follow her up in our clinic.

\section{Discussion}

These two cases demonstrate patients who have developed atherosclerotic disease, despite a supposedly 'protective level' of HDL. They also present with elevated LDL levels. This raises questions regarding our interpretation and management of concurrent lipid abnormalities in the context of a raised HDL level. Observational studies have demonstrated that patients with a lower level of HDL have a higher risk of cardiovascular disease [17]. The Framingham cohort also supported this. The primary hypothesis behind this relationship has been the role of HDL in reverse cholesterol transport and as a result, low HDL is now utilized as an important component of contemporary cardiovascular risk estimation [18]. Contemporary data has established a U-shaped association between HDL and adverse events in numerous population studies, further complicating our understanding of the clinical significance of HDL [19]. This is further emphasized by the prospective study of almost two million US veterans, where a U-shaped relationship between HDL level and all-cause mortality was demonstrated [20]. Likewise, a Canadian study highlighted that people with a HDL level $>90 \mathrm{mg} / \mathrm{dL}$ were associated with high risk of all-cause mortality [21]. In addition, HDL levels $3.5 \mathrm{mmol} / \mathrm{L}$ and $<1.0 \mathrm{mmol} / \mathrm{L}$ were associated with high all-cause mortality and high cardiovascular mortality in two prospective Danish cohorts [22]. According to the observations in the above studies, the twopatients described in our study would be categorized as "high risk" due to their HDL concentration.

More recent analysis has suggested that this relationship may be modulated by other components of the lipid panel. Some studies have suggested that low $\mathrm{HDL}$ is not a truly independent risk factor for ASCVD in patients with normal levels of triglyceride and LDL [23]. The authors propose that while high HDL is protective against ASCVD overall, it must be interpreted in the context of concurrent abnormalities in triglyceride or LDL levels. Before considering HDL function, it is important to initially convey that High HDL levels do not indicate any reduced risk and should not let physicians discount elevated LDL levels. As observed in the above cases with levels of $3.7 \mathrm{mmol} / \mathrm{L}$ and $4.3 \mathrm{mmol} / \mathrm{L}$ respectively, the LDL are high, despite the high the HDL. In this regard it would also be important to abandon ratios. As mentioned earlier, this has important implications as several 
trials have failed to demonstrate a therapeutic benefit from HDL raising therapies [3-5]. We will discuss these trials in more detail later in the article.

\section{Evidence challenging causal role of HDL}

Further evidence challenging the causal relationship between HDL and ASCVD comes from studies showing that in some circumstances, high levels of HDL may be associated with an increased risk of ASCVD. Genetic analyses of over 300,000 people confirmed that a genetic variant, called SCARB1 P376L, was associated with elevated HDL levels [24]. The researchers found that people with the variant had unusually high levels of large HDL particles in their blood. To see whether SCARB1 P376L was associated with heart disease, the team acquired data from nearly 50,000 people with coronary heart disease and about 88,000 controls. They found that those with the variant had a significantly higher risk of heart disease. SCARB1 codes for the major HDL receptor on liver cells, scavenger receptor class BI. In mice, genetic manipulations of this gene had effects opposite from those expected if HDL were protective. Over-expression of the gene lowered HDL levels but reduced atherosclerosis. Deletion raised HDL levels but an increased rate of atherosclerosis. Similarly, Apolipoprotein A-1 Milano is a naturally occurring mutated variant of the apolipoprotein A1 protein found in human HDL. ApoA1 Milano is associated with a significantly reduced incidence of cardiovascular disease, even though it causes a reduction in HDL levels and an increase in triglyceride levels [25]. HDL is a complex particle that is subjected to dynamic remodeling through its interplay with numerous enzymes, tissues, lifestyle and diet, highlighting that the overall understanding of its functions and roles are more intricate than anticipated [7]. These studies provide an intriguing insight into the complexities associated with HDL in vivo. It appears that is not simply a linear relationship with higher HDL levels equating to lower rates of ASCVD.

\section{HDL metabolism}

HDLs as a group of particles can demonstrate intrinsically diverse metabolism and functions depending on their particular proteomic, lipidomic, structural and chemical properties. In addition, HDL carries numerous lipids, proteins, enzymes and micro RNAs, which all may play a functional role. The lipidome of HDL has been shown to possess functional properties [26], while the micro RNA profile of HDL can be altered in pathological conditions [27] further emphasizing the dynamic flow of HDL. The lipidome and proteome of HDLs are transformed, with increased triglycerides and decreased phospholipid. Utilization of contemporary methods of classification, such as nuclear magnetic resonance, has increased interest on expounding the complex nature of lipidome, proteome, and structural difference of HDL particles [26,28-30].

\section{Functional HDL deficiency}

This leads us to the concept of 'functional HDL deficiency'. This refers to cases where, despite normal levels of HDL on quantitative measurement, patients may be functionally deficient. This functional deficiency may result from HDL losing its function and becoming less athero-protective ('loss of function'), or alternatively, HDL may become dysfunctional, resulting in pro-inflammatory effects ('gain of dysfunction').

\section{Defining HDL dysfunction}

Defining functional HDL deficiency or HDL dysfunction has been attempted in several different ways. This can include assessing for oxidative modification of HDL particles and utilizing functional assays. These functional assays can test for, amongst others; macrophage cholesterol efflux and the ability of HDL to inhibit monocyte chemotaxis. Some authors have proposed the concept of an 'inflammatory index' for HDL, which is defined as the relative capacity of HDL to inhibit monocyte chemotaxis induced by oxidized LDL. This monocyte chemotaxis assay has been shown to distinguish between ASCVD and control individuals with higher accuracy than the measurement of HDL levels [31].

Functional assays may not be scalable and feasible at present. An alternative approach may be to assess the HDL proteome in order to identify changes in the protein composition of HDL. These changes may be surrogate markers of altered HDL function. Surrogate markers may include changes in the size/density of HDL particles and the specific proteome present. Identification of these alterations in HDL may confer physicians with a better ability to predict cardiovascular risk. Certain subtypes of HDL have already been identified which seem to predict cardiovascular risk more accurately than HDL alone [32]. We will discuss some of these in the next section.

\section{Apolipoprotein Al}

Apolipoprotein Al (ApoA1) is the major protein component found in HDL. It is considered to play a vital role in the reverse cholesterol transport process as shown in Figure 1. However, it may undergo post-translational modification by myeloperoxidase (MPO), which can impair its function as a cholesterol acceptor. MPO can induce molecular alterations in ApoA1 including nitration and chlorination of specific apoAl tyrosine residues. This is associated with specific loss of ABCA1 mediated cholesterol acceptor activity. As a result apoAl cholesterol acceptor activity is inversely correlated with ApoAI nitro- and chloro-tyrosine levels. Unsurprisingly, plasma ApoAl nitro- and chloro-tyrosine levels are higher in ASCVD patients than in controls and plasma MPO levels have been positively associated with ASCVD and the risk of MACE $[33,34]$. Some authors have proposed that levels of nitro- and chloro-tyrosine in ApoA1 may serve 


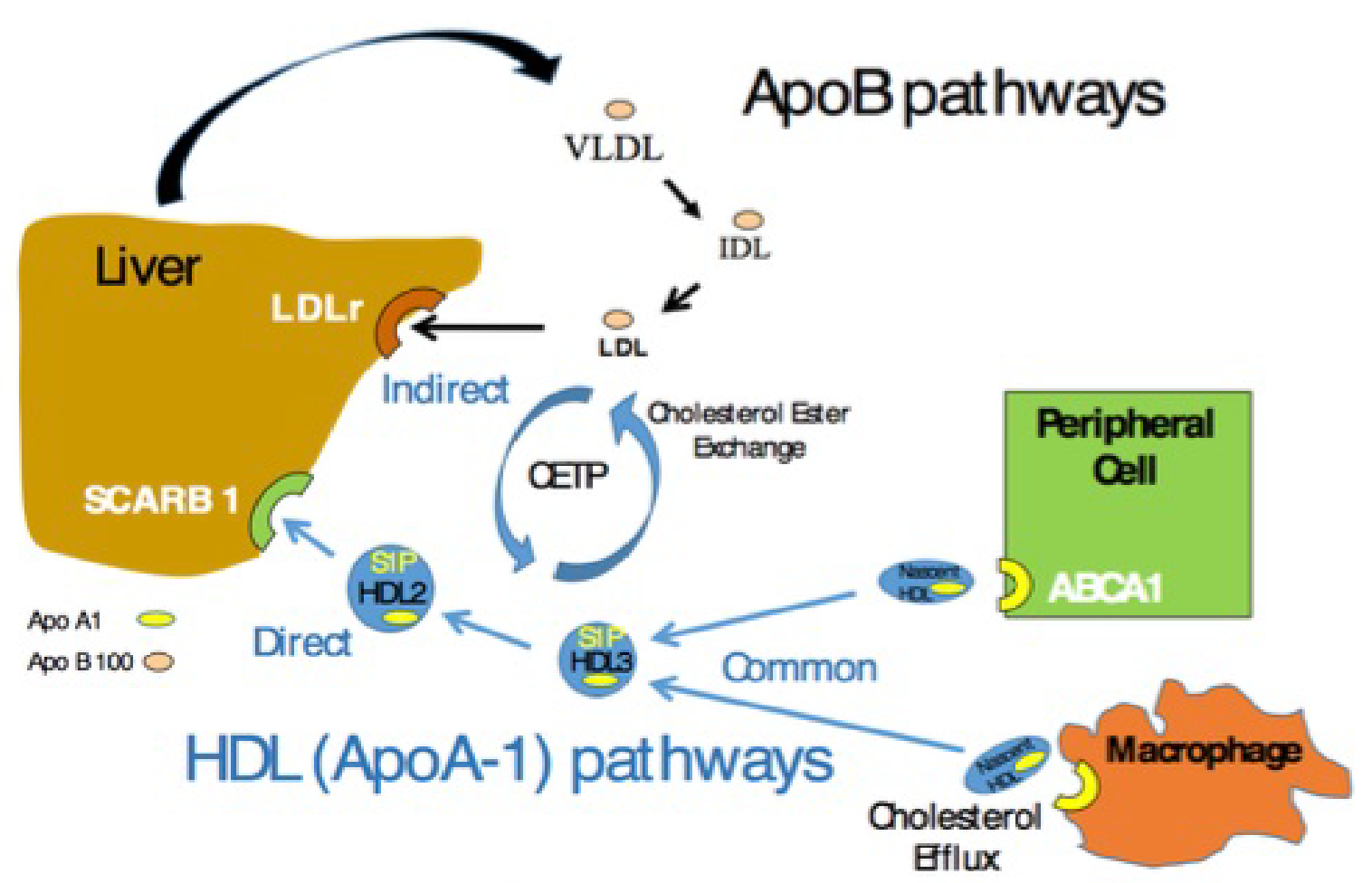

(C) Advanced Lipid Management And Research Centre

Figure 1: ApoA1 pathway and effect on reverse cholesterol transport. Reproduced courtesy of Prof. Vincent Maher of the Advanced Lipid Management and Research (ALMAR) Centre at Tallaght University Hospital

as useful markers of MPO activity in vivo. This oxidative modification may be associated with several conditions; chronic kidney disease, metabolic syndrome and type 2 diabetes mellitus. There have been other modes of oxidative modifications of apoA-I beyond nitration or chlorination documented. In a study by Hazen, et al., Tryptophan modification of apoAl was primarily responsible for the myeloperoxidase-mediated loss of the cholesterol acceptor activity of apoAI [35].

\section{Sphingosine-1-Phosphate}

Sphingosine-1-Phosphate (S1P) is another proposed mechanistic cause for HDL dysfunction. It is a lipid mediator found in the erythrocytes, platelets and endothelial cells, and binds to the lysophospholipid receptor family. Only S1P-bound HDL (60\% of total plasma S1P) seems to be active, whereas albumin-bound S1P (40\% of plasma $\mathrm{S} 1 \mathrm{P})$ acts as a reservoir. One study discovered that HDL oxidation resulted in a $44 \%$ loss of HDL-S1P compared to native HDL [36]. Western blot analysis of apolipoprotein $\mathrm{Al}$ (ApoAI) as the major HDL apolipoprotein and apolipoprotein M (ApoM) as an important S1P binding partner in HDL showed that both proteins were altered by oxidation: ApoM was virtually absent in oxidized HDL, whereas ApoAl appeared as several immunoreactive bands. It has been observed that healthy HDL and ASCVD HDL with the same S1P content are equally efficient. The authors proposed that HDL signaling was mainly dependent on their ability to carry, retain, and present
S1P. Interestingly, this group was subsequently able to artificially increase the S1P content of HDL, thereby increasing its function. This may represent a potential future therapeutic strategy to restore HDL function.

\section{Serum Amyloid A}

Serum Amyloid A (SAA) is an acute phase HDL-associated apolipoprotein produced primarily by hepatocytes [37]. SAA is secreted during the acute phase of inflammation and has several immunomodulatory roles. These include neutrophil activation and cytokine induction [38]. This may play a key role in local and systemic inflammation and free fatty acid production, promoting ASCVD [39]. SAA also enables the production of reactive oxygen species (ROS) through its chemotactic ligand properties and indirect affect on the potent chemotactic factor, fMLP [40]. This may similarly promote the establishment and advancement of ASCVD [41]. A review by Egom, et al. highlights the literature giving weight to the theory that the S1P/SAA Index may affect the HDL athero-protective properties and thus indicate a potential target for therapeutic interventions. Increased SAA was determined to be highly associated with increased all-cause and cardiovascular mortality [42].

Many studies have demonstrated raised serum SAA levels in patients with ASCVD [43]. It has also been shown to be elevated in high cardiovascular risk populations including smokers and patients with diabetes mellitus and chronic kidney disease $[44,45]$. Raised serum 
levels of SAA may inhibit endothelial NO production, increase production of ROS and impair reverse cholesterol transport [43], Patients with ASCVD have also been demonstrated to express considerably higher levels of SAA in HDL particles in comparison to healthy subjects [46]. This accumulation of SAA into HDL particles may disrupt their anti-atherothrombotic mechanisms $[47,48]$, leading to HDL dysfunction $[49,50]$.

Based on the data discussed above, SAA may be considered the 'inflammatory marker' and S1P the 'athero-thrombotic marker' with regard to HDL function. Evaluating both markers concurrently may provide physicians with a comprehensive overview of HDL function.

\section{Apolipoprotein C-III}

Apolipoprotein C-III (ApoC-III) is a pro-inflammatory protein that resides on the surface of very-low-density lipoprotein (VLDL), LDL, and HDL particles. Presence of apoC-III in HDL sub-fractions from plasma may identify participants with an increased risk of future CHD [51]. ApoC-III inhibits lipoprotein lipase and HDL particles bearing ApoC-III are associated with an increased rather than a decreased risk of ASCVD. It has been postulated that the presence/absence of apoC-III in HDL sub-fractions from plasma could be used to stratify an individual's future risk of ASCVD. One study [52] reported that the relative risk of CHD per standard deviation of $\mathrm{HDL}$ without ApoC-III was 0.66 (95\% Cl 0.53-0.93), whereas it was $1.18(95 \% \mathrm{Cl} 1.03-1.34)$ for HDL with ApoC-III; these findings indicate that HDL subtypes without ApoC-III were inversely associated with the risk of $C H D$, whereas HDL subtypes with ApoC-III showed a direct associationwith the risk of CHD. These data suggest that ApoC-III interferes with the atheroprotective function of HDL. With regards to the role of lipases, deLemos, et al. undertook a genome-wide association study that described several variations of the endothelial lipase gene that were exclusively associated with HDL [53]. One specific loss of function of single nucleotide polymorphism (Asn396Ser) was associated with a substantialrise in HDL and reduced endothelial lipase activity in vitro [54] potentially correlating the role of lipases with the U-shaped curve discussed earlier.

\section{HDL raising therapies}

This paradigm shift to a qualitative rather than quantitative assessment of HDL might explain the failure of several HDL raising therapies to demonstrate a reduction in cardiovascular events. This has been particularly noted with the CETP inhibitors, torcetrapib and dalcetrapib. In the ILLUMINATE study [10], Torcetrapib led to an increase in all-cause mortality and cardiovascular events, although it was able to increase HDL by $72 \%$ and decrease LDL by $25 \%$. It should be noted that unfortunately, torcetrapib also increases blood pressure and increases aldosterone as an off-target, non-mechanism-based effect. In the dal-OUTCOMES [11] study, dalcetrapib increased HDL cholesterol levels but did not reduce the risk of recurrent cardiovascular events in patients who had had a recent acute coronary syndrome. These studies provide further insight into HDL function and indicate that a focus on simply raising HDL levels will not be sufficient to improve cardiovascular outcomes. Potential therapeutic avenues in the future may include oxidation resistant $\mathrm{HDL}$, oxidation resistant APoA1 and artificial elevation of S1P in order to restore HDL function.

\section{Final thoughts}

As we can see from this article, the relationship between cardiovascular disease and HDL is complex. This complexity reflects the varied proteome and pleiotropic functions associated with HDL in vivo. It appears that quantification of HDL levels is not always sufficient to predict cardiovascular risk. As can be seen in our two cases, patients can still develop cardiovascular disease despite levels of HDL traditionally thought of as athero-protective.

Much progress has been made with regard to assessment of the HDL proteome and HDL function. The hope is that in the future, this information may translate to improved risk stratification and therapeutic strategies in order to reduce the risk of cardiovascular disease. However much work remains in order to further clarify the optimum diagnostic and therapeutic approach.

In the interim period, physicians should remain cognizant that HDL may not always be atheroprotective. As such HDL levels must not be viewed in isolation and should be interpreted in the context of concomitant lipid abnormalities in order to determine the optimum therapeutic strategy for an individual patient. It seems that with regard to $\mathrm{HDL}$, quality is more important than quantity.

\section{Conclusion}

HDL values need to be interpreted in the context of concomitant lipid abnormalities and the overall risk factor profile of the patient.

High levels of HDL may not always be protective.

In the future, functional testing of HDL may provide further information for physicians to estimate cardiovascular risk and decide on optimum treatment strategies. Future therapeutic modalities may aim to restore and optimize HDL function rather than to simply increase HDL quantity.

\section{Compliance with Ethical Standards}

\section{Funding}

This study received no funding.

\section{Conflict of Interest}

The authors report no relationships that could be construed as a conflict of interest. 


\section{Ethical approval}

All procedures performed in studies involving human participants were in accordance with the ethical standards of the institutional and/or national research committee and with the 1964 Helsinki declaration and its later amendments or comparable ethical standards.

\section{Informed consent}

Informed consent was obtained from all individual participants included in the study.

\section{References}

1. Kannel WB, Dawber TR, Friedman GD, Glennon WE, McNamara PM (1964) Risk factors in coronary heart disease. An evaluation of several serum lipids as predictors of coronary heart disease; The Framingham Study. Ann Intern Med 61: 888-899.

2. Castelli WP, Anderson K, Wilson PW, Levy D (1992) Lipids and risk of coronary heart disease. The Framingham Study. Ann Epidemiol 2: 23-28.

3. Di Angelantonio E, Sarwar N, Perry P, Kaptoge S, Ray KK, et al. (2009) Major lipids, apolipoproteins, and risk of vascular disease. JAMA 302: 1993-2000.

4. Shao B, Heinecke JW (2017) Quantifying HDL proteins by mass spectrometry: How many proteins are there and what are their functions? Expert Rev Proteomics 15: 31-40.

5. Vaisar T, Pennathur S, Green PS, Gharib SA, Hoofnagle AN, et al. (2007) Shotgun proteomics implicates protease inhibition and complement activation in the antiinflammatory properties of HDL. J Clin Invest 117: 746-756.

6. Shiflett AM, Bishop JR, Pahwa A, Hajduk SL (2005) Human high density lipoproteins are platforms for the assembly of multi-component innate immune complexes. J Biol Chem 280: 32578-32585.

7. Jomard A, Osto E (2020) High density lipoproteins: Metabolism, function, and therapeutic potential. Front Cardiovasc Med 7: 39.

8. Holmes MV, Asselbergs FW, Palmer TM, Drenos F, Lanktree MB, et al. (2015) Mendelian randomization of blood lipids for coronary heart disease. Eur Heart J 36: 539-550.

9. The HPS2-THRIVE Collaborative Group, Landray MJ, Haynes R, Hopewell JC, Parish S, Aung T, et al. (2014) Effects of extended-release niacin with laropiprant in highrisk patients. N Engl J Med 371: 203-212.

10. Barter PJ, Caulfield M, Eriksson M, Grundy SM, Kastelein JJ, et al. (2007) Effects of torcetrapib in patients at high risk for coronary events. N Engl J Med 357: 2109-2122.

11. Schwartz GG, Olsson AG, Abt M, Ballantyne CM, Barter PJ, et al. (2012) Effects of dalcetrapib in patients with a recent acute coronary syndrome. N Engl J Med 367: 20892099.

12. Madsen CM, Varbo A, Tybjærg-Hansen A, Frikke-Schmidt R, Nordestgaard BG (2018) U-shaped relationship of HDL and risk of infectious disease: Two prospective population-based cohort studies. Eur Heart J 39: 1181-1190.

13. Nofer JR, van der Giet M, Tolle M, Wolinska I, von Wnuck Lipinski K, et al. (2004) HDL induces NO-dependent vasorelaxation via the lysophospholipid receptor S1P3. J Clin Invest 113: 569-581.

14. Kuhn FE, Mohler ER, Satler LF, Reagan K, Lu DY, et al.
(1991) Effects of high density lipoprotein on acetylcholine induced coronary vasoreactivity. Am J Cardiol 68: 14251430.

15. Nicholls SJ, Lundman P, Harmer JA, Cutri B, Griffiths KA, et al. (2006) Consumption of saturated fat impairs the anti-inflammatory properties of high-density lipoproteins and endothelial function. J Am Coll Cardiol 48: 715-720.

16. Yuhanna IS, Zhu Y, Cox BE, Hahner LD, Osborne-Lawrence S, et al. (2001) High-density lipoprotein binding to scavenger receptor-BI activates endothelial nitric oxide synthase. Nat Med 7: 853-857.

17. Miller GJ, Miller NE (1975) Plasma-high-density-lipoprotein concentration and development of ischaemic heart-disease. Lancet 1: 16-19.

18. D'Agostino RB Sr, Vasan RS, Pencina MJ, Wolf PA, Cobain M, et al. (2008) General cardiovascular risk profile for use in primary care: The Framingham Heart Study. Circulation 117: 743-753.

19. Marc P Allard-Ratick, Pratik Sandesara, Arshed A Quyyumi, Laurence S Sperling (2019) Everything in moderation: Investigating the U-shaped link between hdl cholesterol and adverse outcomes. US Cardiology Review 13: 49-53.

20. Bowe B, Xie Y, Xian H, Balasubramanian S, Zayed MA, et al. (2016) High density lipoprotein cholesterol and the risk of all-cause mortality among US veterans. Clin J Am Soc Nephrol 11: 1784-1793.

21. Ko DT, Alter DA, Guo H, Koh M, Lau G, et al. (2016) High-density lipoprotein cholesterol and cause-specific mortality in individuals without previous cardiovascular conditions: The CANHEART study. J Am Coll Cardiol 68: 2073-2083.

22. Madsen CM, Varbo A, Nordestgaard BG (2017) Extreme high high-density lipoprotein cholesterol is paradoxically associated with high mortality in men and women: Two prospective cohort studies. Eur Heart J 38: 2478-2486.

23. Bartlett J, Predazzi IM, Williams SM, Bush WS, Kim Y, et al. (2016) Is isolated low high-density lipoprotein cholesterol a cardiovascular disease risk factor? New insights from the framingham offspring study. Circ Cardiovasc Qual Outcomes 9: 206-212.

24. Zanoni P, Khetarpal SA, Larach DB, Hancock-Cerutti WF, Millar JS, et al. (2016) Rare variant in scavenger receptor $\mathrm{BI}$ raises $\mathrm{HDL}$ cholesterol and increases risk of coronary heart disease. Science 351: 1166-1171.

25. Alexander ET, Tanaka M, Kono M, Saito H, Rader DJ, et al. (2009) Structural and functional consequences of the Milano mutation (R173C) in human apolipoprotein A-I. J Lipid Res 50: 1409-1419.

26. Kontush A, Lhomme M, Chapman MJ (2013) Unraveling the complexities of the HDL lipidome. J Lipid Res 54: 29502963.

27. Vickers KC, Palmisano BT, Shoucri BM, Shamburek RD, Remaley AT (2011) MicroRNAs are transported in plasma and delivered to recipient cells by high-density lipoproteins. Nat Cell Biol 13: 423-433.

28. Otvos JD, Jeyarajah EJ, Bennett DW, Krauss RM (1992) Development of a proton nuclear magnetic resonance spectroscopic method for determining plasma lipoprotein concentrations and subspecies distributions from a single, rapid measurement. Clin Chem 38: 1632-1638.

29. O'Reilly M, Dillon E, Guo W, Finucane O, McMorrow A, et al. (2016) High-density lipoprotein proteomic composition, 
and not efflux capacity, reflects differential modulation of reverse cholesterol transport by saturated and monounsaturated fat diets. Circulation 133: 1838-1850.

30. Kajani S, Curley S, McGillicuddy FC (2018) Unravelling HDL-looking beyond the cholesterol surface to the quality within. Int J Mol Sci 19: E1971.

31. Ansell BJ, Navab M, Hama S, Kamranpour N, Fonarow G, et al. (2003) Inflammatory/antiinflammatory properties of high-density lipoprotein distinguish patients from control subjects better than high-density lipoprotein cholesterol levels and are favorably affected by simvastatin treatment. Circulation 108: 2751-2756.

32. Parra ES, Panzoldo NB, Zago VH, Scherrer DZ, Alexandre F, et al. (2014) HDL size is more accurate than HDL cholesterol to predict carotid subclinical atherosclerosis in individuals classified as low cardiovascular risk. PLoS One 9: e114212.

33. Zhang R, Brennan ML, Fu X, Aviles RJ, Pearce GL, et al. (2001) Association between myeloperoxidase levels and risk of coronary artery disease. JAMA 286: 2136-2142.

34. Meuwese MC, Stroes ES, Hazen SL, vanMiert JN, Kuivenhoven JA, et al. (2007) Serum myeloperoxidase levels are associated with the future risk of coronary artery disease in apparently healthy individuals: The EPIC-Norfolk prospective population study. J Am Coll Cardiol 50: 159-165.

35. Peng DQ, Brubaker G, Wu Z, Zheng L, Willard B, et al (2008) Apolipoprotein A-I tryptophan substitution leads to resistance to myeloperoxidase-mediated loss of function. Arterioscler Thromb Vasc Biol 28: 2063-2070.

36. Settler K, Graler M, Keul P, Weske S, Reimann CM, et al. (2015) Defects of high-density lipoproteins in coronary artery disease caused by low Sphingosine-1-Phosphate Content: Correction by Sphingosine-1-Phosphate-Loading. J Am Coll Cardiol 66: 1470-1485.

37. Han CY, Tang C, Guevara ME, Wei H, Wietecha T, et al (2016) Serum amyloid A impairs the antiinflammatory properties of HDL. J Clin Invest 126: 266-281.

38. Uslu AU, Aydin B, Icagasıoğlu IS, Balta S, Deveci K, et al. (2016) The relationship among the level of serum amyloid $\mathrm{A}$, high-density lipoprotein and microalbuminuria in patients with familial mediterranean fever. J Clin Lab Anal 30: 10031008.

39. Yang RZ, Lee MJ, Hu H, Pollin TI, Ryan AS, et al. (2006) Acute-phase serum amyloid A: An inflammatory adipokine and potential link between obesity and its metabolic complications. PLoS Medicine 3: e287.

40. Su SB, Gong W, Gao JL, Shen W, Murphy PM, et al. (1999) A seven-transmembrane, $G$ protein-coupled receptor, FPRL1, mediates the chemotactic activity of serum amyloid A for human phagocytic cells. J Exp Med 189: 395-402.

41. Bae YS, Yi HJ, Lee HY, Jo EJ, Im Kim J, et al. (2003) Differential activation of formyl peptide receptor-like 1 by peptide ligands. J Immunol 171: 6807-6813.
42. Egom EE, Shiwani HA, Pharithi RB, Canning R, Khan B, et al. (2018) Dynamic changes of the composition of plasma HDL particles in patients with cardiac disease: Spotlight on sphingosine-1-phosphate/serum amyloid A ratio. Clin Exp Pharmacol Physiol 45: 319-325.

43. Zewinger S, Drechsler C, Kleber ME, Dressel A, Riffel J, et al. (2015) Serum amyloid A: High-density lipoproteins interaction and cardiovascular risk. Eur Heart J 36: 3007-3016.

44. Holzer M, Birner-Gruenberger R, Stojakovic T, El-Gamal D, Binder V, et al. (2011) Uremia alters HDL composition and function. J Am Soc Nephrol 22: 1631-1641.

45. Griffiths K, Pazderska A, Ahmed M, McGowan A, Maxwell AP, et al. (2017) Type 2 diabetes in young females results in increased serum amyloid $A$ and changes to features of high density lipoproteins in both $\mathrm{HDL}_{2}$ and $\mathrm{HDL}_{3}$. J Diabetes Res 2017: 1314864.

46. Wang DX, Liu H, Yan LR, Zhang YP, Guan XY, et al. (2013) The relationship between serum amyloid $A$ and apolipoprotein $A-I$ in high-density lipoprotein isolated from patients with coronary heart disease. Chin Med J 126: 3656-3661.

47. Coetzee GA, Strachan AF, Van Der Westhuyzen DR, Hoppe HC, Jeenah MS, et al. (1986) Serum amyloid A-containing human high density lipoprotein 3 . Density, size, and apolipoprotein composition. J Biol Chem 261: 9644-9651.

48. Van Lenten BJ, Navab M, Shih D, Fogelman AM, Lusis AJ (2001) The role of high-density lipoproteins in oxidation and inflammation. Trends Cardiovasc Med 11: 155-161.

49. Korita I, Bulo A, Langlois MR, Verhoye E, Blaton V (2013) Serum amyloid $A$ is independently related to apolipoprotein A-I but not to HDL-cholesterol in patients with angina pectoris. Clin Biochem 46: 1660-1663.

50. Ansell BJ, Watson KE, Fogelman AM, Navab M, Fonarow GC (2005) High-density lipoprotein function recent advances. J Am Coll Cardiol 46: 1792-1798.

51. Onat A, Hergenc G, Sansoy V, Fobker M, Ceyhan K, et al. (2003) Apolipoprotein C-III, a strong discriminant of coronary risk in men and a determinant of the metabolic syndrome in both genders. Atherosclerosis 168: 81-89.

52. Jensen MK, Rimm EB, Furtado JD, Sacks FM (2012) Apolipoprotein C-III as a potential modulator of the association between HDL-cholesterol and incident coronary heart disease. J Am Heart Assoc 1.

53. deLemos AS, Wolfe ML, Long CJ, Sivapackianathan R, Rader DJ (2002) Identification of genetic variants in endothelial lipase in persons with elevated high-density lipoprotein cholesterol. Circulation 106: 1321-1326.

54. Edmondson AC, Brown RJ, Kathiresan S, Cupples LA, Demissie S, et al. (2009) Loss-of-function variants in endothelial lipase are a cause of elevated HDL cholesterol in humans. J Clin Invest 119: 1042-1050. 